\title{
Écritures dramatiques, dossier a cura di Musanji Ngalasso-Mwatha
}

\section{Carminella Biondi}

\section{(2) OpenEdition}

1 Journals

\section{Edizione digitale}

URL: http://journals.openedition.org/studifrancesi/6116

DOI: $10.4000 /$ studifrancesi.6116

ISSN: 2421-5856

\section{Editore}

Rosenberg \& Sellier

\section{Edizione cartacea}

Data di pubblicazione: 1 mai 2011

Paginazione: 219-220

ISSN: 0039-2944

\section{Notizia bibliografica digitale}

Carminella Biondi, «Écritures dramatiques, dossier a cura di Musanji Ngalasso-Mwatha», Studi Francesi [Online], 163 (LV | I) | 2011, online dal 30 novembre 2015, consultato il 09 janvier 2021. URL: http:// journals.openedition.org/studifrancesi/6116 ; DOI: https://doi.org/10.4000/studifrancesi.6116

Questo documento è stato generato automaticamente il 9 janvier 2021.

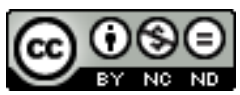

Studi Francesi è distribuita con Licenza Creative Commons Attribuzione - Non commerciale - Non opere derivate 4.0 Internazionale. 


\title{
Écritures dramatiques, dossier a cura di Musanji Ngalasso-Mwatha
}

\author{
Carminella Biondi
}

\section{NOTIZIA}

Écritures dramatiques, dossier a cura di Musanji NGALASSO MWATHA, «Présence francophones», n. 73, 2009, pp. 5-109.

1 La presentazione si apre con una domanda non retorica come ci si rende subito conto leggendo l'intera presentazione e i saggi contenuti nel dossier: «Que faut-il entendre par écriture dramatique?». E la risposta del curatore è tutt'altro che scontata e, bisogna dirlo, tutt'altro che convincente, perché implica una vastissima gamma di situazioni che vanno dal dramma inteso in senso classico, passando per la scrittura drammatica in un testo narrativo, fino alle «affres du style» (p. 6), a quella sofferenza creativa che ha, per intenderci, il suo maestro in Flaubert: «C'est sur cette idée de l'atroce du style et du drame de l'écriture que nous voudrions fonder la réflexion qui nourrit les articles contenus dans le présent volume [...]. Les auteurs tentent d'interroger la spécificité de la parole agissante du théâtre, tout en recherchant les modes d'imprégnation de l'écriture dramatique dans les autres genres littéraires» (p. 7).

2 Seguono otto saggi che trattano una serie di problemi importanti concernenti le letterature francofone, in particolare africane, ma anche la lettura più attenta fatica a farli entrare senza qualche piccola forzatura nel quadro di riferimento già di per sé così elastico. Certo il primo saggio di Salom KOMIAN GABNOU è focalizzato su una tematica pertinente: Théâtres du roman: les scènes de l'écriture francophone (pp. 11-22), ma già il sottotitolo indica un campo così sterminato da lasciare quantomeno perplessi, come del resto la conclusione: «On remarque que le malicieux rapport que l'écrivain francophone entretient avec une langue, dont généralement il a une parfaite maîtrise, le place exemplairement dans la posture du régisseur et lui permet de s'affranchir du code d'une langue à tous, exactement comme au théâtre où chaque mise en scène est 
unique et singulière» (p. 21). Un discorso diverso va fatto per il saggio che segue di Bernard MOURALIS, Le roman africain: drame ou histoire?, in cui vengono effettivamente esaminati i rapporti intercorrenti fra il genere teatrale e quello narrativo nel romanzo africano, un romanzo caratterizzato da alcune peculiarità che possono giustificare il confronto.

3 Il terzo saggio di Savrina PARevadee Chinine su L'art de l'«écrire» chez Patrick Chamoiseau (pp. 36-45), colloca il problema della scrittura del romanziere martinicano all'interno di un discorso più generale che concerne la letteratura postcoloniale: «Ainsi l' 'écrire' par sa structure narrative et ses thématiques récurrentes, est un travail de style recherché, entraînant 'un labeur atroce' et une 'souffrance indicible', et correspond à la logique d'un mouvement littéraire qui a pour but de désaliéner, décoloniser et libérer» (p. 44). Una conclusione piuttosto sorprendente!

4 Seguono alcuni saggi di sicuro interesse per le tematiche che affrontano, anche se, come ho detto, non è facile trovarvi un legame cogente con l'assunto: Lila KERMAS su L'écriture de la perte chez Assia Djebar (pp. 46-54), Jean-Fernand BÉDIA; Le "français de rue» et l'écriture de la guerre: portée et signification (pp. 55-65); Virginie DARRIET-FÉRÉOL, De la parole poétique à la parole politique dans les ceuvres d'Aimé Césaire et de Sony Labou Tansi (pp. 66-75); Omar FERTAT, Le théâtre amateur marocain. Trajectoire d'un théâtre alternatif (pp. 76-91); Pierre FANDIO, Mutations politiques et processus de légitimation culturelle: considérations sur le théâtre populaire camerounais (pp. 92-109). 